\title{
Análise e incidência do melanoma cutâneo em um hospital de câncer localizado no Triângulo Mineiro
}

\section{Analysis and incidence of skin melanoma in a cancer hospital located in the Triângulo Mineiro}

Análisis e incidencia del melanoma cutáneo em un hospital de cáncer situado en el

Recebido: 12/05/2017

Aprovado: 04/11/2017

Publicado: 07/05/2018 Triángulo Mineiro

\section{Ricardo Dias da Silva1 Meyre Aparecida Inácio Dias ${ }^{2}$}

Este estudo teve por objetivo avaliar o melanoma cutâneo a partir da incidência e das variáveis: gênero, cor da pele, idade, localização do tumor e tipo histológico. Esta é uma pesquisa descritiva, quantitativa, retrospectiva e, de corte transversal. Foram avaliadas 81 biópsias de pacientes atendidos em consulta médica em um hospital de câncer da cidade de Patrocínio, MG, entre janeiro de 2015 e maio de 2016. Encontrou-se 42 biópsias com carcinoma basocelular (51,85\%), 20 com carcinoma espinocelular $(24,69 \%), 3$ com melanoma (3,7\%) e, 16 com outros diagnósticos e resultados inconclusivos $(19,75 \%)$. O melanoma foi o de menor incidência entre as neoplasias de pele; predominante em pessoas de pele clara; variável gênero depende da população estudada; o melanoma extensivo superficial é o tipo predominante; e, a variável idade mostrando-se controversa. A pesquisa propiciou informações sobre o câncer de pele numa região do estado de Minas Gerais e pode ser um sensor para a inclusão de ações de saúde sobretudo, nas de prevenção e de detecção precoce.

Descritores: Melanoma; Neoplasias cutâneas; Epidemiologia.

This study aimed to evaluate the cutaneous melanoma from the incidence and the variables: gender, color of skin, age, location of the tumor and histologic type. This is a descriptive, quantitative research, retrospective and cross-sectional. We evaluated 81 biopsies of patients in medical consultation in a cancer hospital in the city of Patrocínio, MG, Brazil, between January 2015 and May 2016. We found 42 biopsies with basal cell carcinoma (51.85\%), 20 with squamous cell carcinoma (24.69\%), 3 with melanoma (3.7\%) and 16 with other diagnoses and inconclusive results (19.75\%). The melanoma was lower between the skin neoplasms; prevalent in light-skinned people; gender variable depends on the population studied; the superficial spreading melanoma is the predominant type; and the variable age is controversial. The research provided information on skin cancer in a region of Minas Gerais State in the Southeast region of Brazil and can be a sensor for the inclusion of health actions especially in the prevention and early detection.

Descriptors: Melanoma; Skin neoplasms; Epidemiology.

Este estudio tuvo por objetivo evaluar el melanoma cutáneo a partir de la incidencia y de las variables: género, color de la piel, edad, localización del tumor y tipo histológico. Esta es una investigación descriptiva, cuantitativa, retrospectiva yde corte transversal. Fueron evaluadas 81 biopsias de pacientes atendidos en consulta médica en un hospital de cáncer de la ciudad de Patrocínio, MG, entre enero de 2015 y mayo de 2016. Se encontraron 42 biopsias con carcinoma baso celular $(51,85 \%), 20$ con carcinoma espino-celular (24,69\%), 3 con melanoma (3,7\%) y, 16 con otros diagnósticos y resultados inconclusos $(19,75 \%)$. El melanoma fue el de menor incidencia entre las neoplasias de piel; predominante en personas de piel clara; variable del género depende de la población estudiada; el melanoma extensivo superficial es el tipo predominante; $y$, la variable edad mostrándose controversia. La investigación propició informaciones sobre el cáncer de piel en una región del estado de Minas Gerais y puede ser un sensor para la inclusión de acciones de salud sobre todo, en las de prevención y de detección precoz.

Descriptores: Melanoma, Neoplasias cutáneas, Epidemiología.

${ }^{1}$ Enfermeiro. Especialista em Oncologia. Especialista em Saúde Coletiva. Especializando em Docência do Ensino Superior pela Universidade Cruzeiro do Sul. Mestrando em Atenção à Saúde pela Universidade Federal do Triângulo Mineiro. Enfermeiro Sanitarista da Secretaria Municipal de Saúde de Patrocínio, MG, Brasil. ORCID: 0000-0002-5856-9318 E-mail: ricardodiv@yahoo.com.br

2 Enfermeira. Especialista em Enfermagem Oncológica. Enfermeira do Hospital do Câncer Dr. José Figueiredo. Patrocínio, MG, Brasil. ORCID: 0000-0003-3436-821X E-mail: meyreapenf@gmail.com 


\section{INTRODUÇÃO}

$\mathbf{0}$ câncer de pele configura-se como o mais frequente em todas as populações ${ }^{1}$. No Brasil, a neoplasia maligna da pele corresponde a $30 \%$ de todos os tumores malignos registrados ${ }^{2}$. As neoplasias cutâneas malignas se apresentam basicamente sob duas formas: o melanoma e o não melanoma ${ }^{1}$.

0 câncer de pele não melanoma (CPNM) é o mais incidente, dividindo-se em: carcinoma basocelular (CBC) e carcinoma espinocelular (CEC) ${ }^{1,2}$. O CBC (70\%) é mais incidente que o CEC (25\%) no Brasil ${ }^{3}$. O prognóstico dessas neoplasias é bom quando o diagnóstico e tratamento acontecem precocemente - alta incidência e baixa mortalidade ${ }^{4}$.

0 melanoma cutâneo maligno (MC) é o câncer de pele mais agressivo, porém é menos incidente que o CPNM $^{1,2,5.0}$ melanoma representa apenas $4 \%$ das neoplasias malignas da pele, apesar de ser o mais grave devido à sua alta capacidade de desenvolvimento de metástase ${ }^{3}$.

Segundo a Estimativa 2016: incidência de câncer no Brasil, do Instituto Nacional do Câncer (INCA), são esperados para o ano de 2016, 5.670 casos de MC (3.000 em homens e 2.670 em mulheres) e 175.760 casos de CPNM no Brasil ${ }^{1}$. Para o estado de Minas Gerais são esperados 330 casos de MC, estando abaixo dos estados de São Paulo, Rio Grande do Sul, Paraná, Santa Catarina e Rio de Janeiro, que possuem as maiores estimativas no país ${ }^{1}$.

Em 2013 ocorreram 1.547 mortes por melanoma no Brasil, sendo 903 homens e 644 mulheres. Os homens são os mais acometidos pelo $\mathrm{MC}^{1,2,6}$. A incidência do $\mathrm{MC}$ vem crescendo nas últimas décadas ${ }^{6-9}$.

No período compreendido entre 460 a 375 a.C. surgiu o primeiro relato referente ao melanoma por Hipócrates; somente em 1838 o termo melanoma foi empregado de forma fidedigna por Robert Carswell na descrição de lesões malignas pigmentadas da pele ${ }^{8}$. Entre os anos 50 e 60 diversos pesquisadores tentavam listar os fatores prognósticos do melanoma8. "Em 1969 Clark e colaboradores aprimoraram o sistema de micro estadiamento utilizando como critério os níveis de invasão da pele"8. Segundo Wainstein e Belfort, "Breslow demonstrou a importância da espessura do melanoma primário"8. Os dois parâmetros, o de Clark e Breslow são utilizados no manejo clínico do melanoma atualmente ${ }^{8}$.

O melanoma cutâneo é um tipo de câncer de pele que tem origem nos melanócitos, com predominância em adultos brancos ${ }^{1,6-9}$. Existem três tipos de células produtoras de melanina, responsáveis por lesões pigmentares: melanócitos epidérmicos; melanócitos dérmicos; e células névicas ${ }^{6}$. Os melanócitos são células pigmentadas localizadas na camada basal da epiderme, infundíbulo e na região bulbar dos folículos pilosos, na coróide (intra-ocular) e em leptomeninges ${ }^{7}$. Essas células são responsáveis pela produção da melanina, que é o principal fator protetor da radiação ultravioleta (RUV) para o tecido cutâneo ${ }^{10-12}$.

Existem quatro tipos de MC descritos na literatura: melanoma extensivo superficial (MES), que corresponde a $70 \%$ dos casos e tem distribuição igualitária em ambos os gêneros; melanoma nodular (MN), acometendo de $10 \%$ a $15 \%$ dos diagnósticos; melanoma lentiginoso acral (MLA), sendo o mais comum em afrodescentes e asiáticos e o responsável por $2 \%$ a $8 \%$ em indivíduos brancos e $30 \%$ a $60 \%$ em não caucasianos; melanoma lentigo maligno (MLM), é raro e, comum em idosos e atingindo $4 \%$ dos $\operatorname{casos}^{6,8,13}$.

0 melanoma é predominantemente cutâneo, porém pode surgir em outras áreas: mucosas, olhos, meninges e outros ${ }^{6,9}$. As principais localizações do MC segundo o tipo histológico são: MES - dorso em homens e membros inferiores em mulheres; $\mathrm{MN}$ - dorso, cabeça e pescoço; MLA -palmoplantar, extremidades digitais, peri e subungueal; MLM - cabeça, pescoço, dorso e mãos 9 . O MC caracteriza-se principalmente por ser uma lesão assimétrica, com bordas irregulares, alteração de coloração e diâmetro maior que 6 $\mathrm{mm}^{8}$.

Com relação à etnia, a incidência do MC é predominante nas pessoas de pele clara (fototipos de Fitzpatrick I e II) ${ }^{13}$. A classificação de Fitzpatrick ocorre através de 
seis fototipos de pele: I, II, III, IV, V e VI; os fototipos I e II caracterizam-se por pele, olhos e cabelos claros que se queimam ao invés de se bronzear ${ }^{14}$. 0 melanoma pode ocorrer em todas as raças, porém com menor incidência em negros ${ }^{9}$. Na população negra, a localização mais comum do MC é a palmoplantar ${ }^{9}$.

Dentre os fatores de risco para o melanoma maligno encontram-se: genética, história familiar de melanoma, gênero (mulheres abaixo de 40 anos e homens acima de 50 anos), exposição solar intermitente (principalmente em caucasianos), fototipo cutâneo (Fitzpatrick I e II), nevos preexistentes (quantitativa: mais de 50 nevos adquiridos; qualitativa: presença de nevos atípicos), imunossupressão e fototerapia com UVA e UVB (em menor grau) ${ }^{6,7,9,13,15-17}$. O MC incide mais em regiões em proximidade a linha do Equador'.

A exposição solar é o fator de risco mais importante para o desenvolvimento do melanoma, responsável por $80 \%$ dos casos, principalmente em pessoas de pele clara e com múltiplas sardas ${ }^{1}$. Os nevos preexistentes são responsáveis de forma significativa pela incidência de $\mathrm{MC}^{6,9}$. Segundo a Estimativa 2016: incidência de câncer no Brasil, do INCA, os nevos melanocíticos prévios são responsáveis por $20 \%$ a $30 \%$ dos casos de melanoma ${ }^{1}$.

As lesões melonocíticas devem ser examinadas quanto a regra $\mathrm{ABCDE}$, onde: $\mathrm{A}$ : assimetria; B: bordas irregulares; C: coloração heterogênea; D: diâmetro superior a $6 \mathrm{~mm}$; E: expansão (modificação do aspecto da lesão) ${ }^{6,7,15,17}$.

Em caso de diagnóstico confirmado de MC, adota-se a Classificação de Breslow, pela qual a lesão é classificada por espessura em cinco graus (de I a V; de $0 \mathrm{~mm}$ a além de 4 $\mathrm{mm})$. A classificação Breslow é a mais importante para estabelecer a conduta terapêutica, o risco de recidiva e o prognóstico ${ }^{6,9,13}$. Outra classificação utilizada é a de Clark, onde o tumor é avaliado pela localização nas camadas da pele (epiderme, derme e hipoderme), possuindo graus de I a $\mathrm{V}^{6}$. A profundidade do tumor (invasão da derme) é também um fator prognóstico importante ${ }^{18}$.
0 melanoma é caracterizado pelo seu potencial de desenvolver metástases e consequente letalidade ${ }^{6,7,9}$. "0 melanoma cutâneo apresenta disseminação por via linfática precoce e,os linfonodos regionais são o sítio mais comum de metástases ${ }^{13}$."

O diagnóstico precoce e tratamento adequado do MC conferem bom prognóstico, sendo essas lesões consideradas de fácil diagnóstico ${ }^{1,13}$. Já os melanomas diagnosticados tardiamente apresentam pior prognóstico ${ }^{1}$. Dos óbitos ocorridos por neoplasias da pele, o MC é responsável por 79\%, sendo muito relevante estudos e ações de promoção, prevenção, diagnóstico precoce, tratamento e reabilitação para esta importante doença ${ }^{13}$.

Assim, maior atenção deve ser dada a detecção e tratamento precoce para o $\mathrm{MC}^{18}$. Em pesquisa realizada no interior do Estado de São Paulo, em um município com 130.000 habitantes, demonstrou-se eficácia nos resultados alcançados na prevenção primária e detecção precoce do MC, mostrando que as ações podem ser reproduzidas em outras cidades brasileiras ${ }^{18}$. Sendo o câncer é um problema de saúde pública no Brasil, tal fato exige que ações de promoção, prevenção, diagnóstico precoce e tratamento adequado sejam realizadas pelos serviços de saúde ${ }^{3,18}$.

Este estudo teve por objetivo avaliar o MC a partir da incidência e das variáveis gênero, cor da pele, idade, localização do tumor e tipo histológico.

\section{MÉTODO}

Trata-se de um estudo descritivo, quantitativo, retrospectivo de corte transversal. Foram avaliadas 81 biópsias anatomopatológicas diagnósticas cutâneas extraídas dos prontuários de pacientes atendidos em um hospital de câncer (HC), da cidade de Patrocínio, localizado na região do Alto Paranaíba/Triângulo Mineiro, no interior do estado de Minas Gerais.

O município tem uma população de 89.333 habitantes e o Índice de Desenvolvimento Humano (IDH) de 0,72919,20. A economia do município é predominantemente no setor agropecuário. 
O critério para a avaliação das biópsias cutâneas foi baseado na realização de consulta médica pelos usuários no Hospital do Câncer Dr. José Figueiredo no período de tempo compreendido entre janeiro de 2015 a maio de 2016. Os critérios de inclusão para os pacientes estarem na pesquisa foram: realização de biópsia cutânea anatomopatológica independente do diagnóstico; realização de tratamento na instituição onde ocorreu o estudo, realização de consulta médica na instituição entre os meses de janeiro de 2015 a maio de 2016.

A partir dos exames com diagnóstico de MC e informações complementares do cadastro e prontuário foram analisadas no estudo as seguintes variáveis: diagnóstico de MC; gênero; cor da pele; idade; localização do tumor e características histológicas. Os achados das biópsias foram divididos em MC, CBC, CEC e outros diagnósticos / resultados inconclusivos, isso com o intuito de contabilizar a incidência do MC frente aos outros diagnósticos.

Os dados foram processados e gerenciados no software Excel® versão 2007, com a elaboração de tabelas para análise das variáveis. Foram elaboradas tabelas para análise de incidência, característica histológica do tumor, gênero, cor da pele e localização do tumor.

Foi realizado teste não paramétrico de Qui-quadrado de Pearson para avaliação da incidência do MC, CBC e CEC, de forma isolada da amostragem total, sendo o nível de significância $(\alpha)$ estabelecido em $5 \%$ e o grau de liberdade (g.l.) em 2.

Para a coleta dos dados e delineamento do estudo foi solicitada por escrito, em duas vias, a autorização prévia da instituição pela autoridade competente, mediante entrega de termo de livre consentimento e esclarecido (TCLE). Essa pesquisa foi autorizada pela instituição detentora dos registros dos usuários - HC Patrocínio - MG. A privacidade de identidade dos usuários foi garantida durante todo processo da pesquisa. 0 estudo está adequado aos critérios da Resolução 466/2012 do Conselho Nacional de Saúde.

\section{RESULTADOS}

Encontrou-se no estudo os diagnósticos de MC, CBC, CEC e outros diagnósticos / resultados inconclusivos. Realizando análise isolada da incidência das neoplasias de pele nos exames avaliados (sem a presença dos outros diagnósticos e resultados inconclusivos encontrados), têm-se um total de 65 biópsias das 81 analisadas, constatando-se: 42 casos de CBC $(64,6 \%), 20$ casos de CEC $(30,76 \%)$ e 3 casos MC $(4,61 \%)$ (Gráfico 1). 0 Teste de Qui-quadrado de Pearson aplicado a essa amostragem, $\operatorname{com} \alpha$ $5 \%$ e g.l. 2 atingiu o valor de 1,195 ( $<<0,05)$.

Gráfico 1.Incidência dos diagnósticos de MC, CBC e CEC entre janeiro de 2015 e maio de 2016. Hospital do Câncer de Dr. José Figueiredo. Patrocínio / MG.

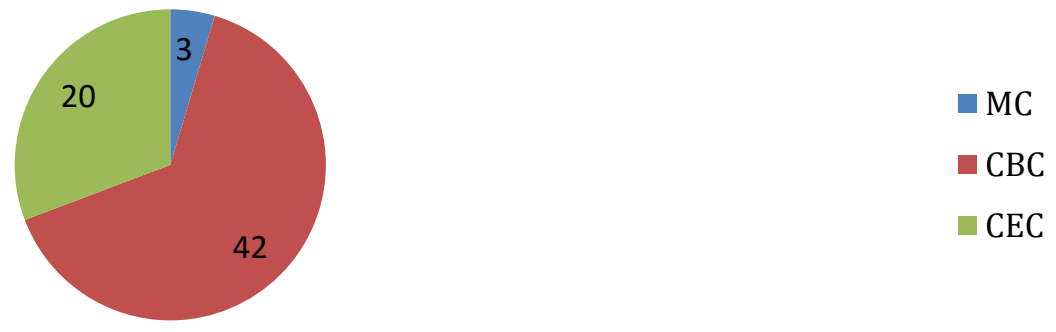

Foram detectadas 42 biópsias com diagnóstico CBC (51,85\%), 20 com CEC $(24,69 \%), 3$ com MC $(3,7 \%)$ e 16 com outros diagnósticos e resultados inconclusivos (19,75\%). O MC mostrou incidência menor do que os CPNM. A incidência do CBC mostrou-se maior do que do CEC.
Para o MC, quanto ao gênero: 2 mulheres $(2,46 \%)$ e 1 homem $(1,46 \%)$. Ocorreu maior quantidade de mulheres com diagnóstico de CBC e CEC $(69,4 \%)$ do que de homens $(29,6 \%)$. Especificamente para o CBC foram encontrados 10 homens $(23,8 \%)$ e 32 mulheres $(76,2 \%)$. No caso do CEC, foram 4 
homens (20\%) e 16 mulheres (60\%). São 14 CNPM em homens e 48 CPNM em mulheres encontrados no estudo (Tabela 1).

Tabela 1. Incidência dos diagnósticos de CBC, CEC, MC e outros diagnósticos conforme gênero entre janeiro de 2015 e maio de 2016. Hospital do Câncer Dr. José Figueiredo. Patrocínio/MG.

\begin{tabular}{lllllllcr} 
Sexo & CBC & \% & CEC & \% & MC & \% & Outros diagnósticos & \% \\
\hline Feminino & 32 & 39,51 & 16 & 19,75 & 2 & 2,46 & 10 & 12,34 \\
Masculino & 10 & 12,34 & 4 & 4,94 & 1 & 1,23 & 6 & 7,41 \\
Resultados & 42 & 51,85 & 20 & 24,69 & 3 & 3,7 & 16 & 19,75 \\
\hline
\end{tabular}

Para a variável idade dentre os melanomas foram identificadas as idades: 42 , 44 e 68 anos, sendo duas mulheres (42 e 68 anos) e um homem ( 44 anos). Para os 3 casos de melanoma a média de idade foi de 51,3 anos; média de 56 anos para as mulheres e 42 anos para o homem. A média de idade para o CBC foi de 64 anos; 63,58 anos para o sexo feminino e 65,30 anos para o sexo masculino. Entre os diagnósticos de CEC, a média de idade geral encontrada foi de 64,80 anos; para mulheres foi 64,81anos e para homens de 64,75 anos dentre os acometidos com o CEC.

Referente à idade encontrou-se para as neoplasias da pele de forma geral no sexo masculino, com a variável idade, encontrou-se 4 homens entre 40 e 49 anos (6,15\%), 2 homens entre os 50 e 59 anos (3,07\%), 3 entre os 60 e 69 anos $(4,61 \%)$ e 6 homens com 70 anos e mais $(9,23 \%)$. Já para o sexo feminino foram 2 mulheres entre 30 e 39 anos $(3,07 \%)$, 8 mulheres entre 40 e 49 anos (12,30\%), 12 mulheres entre 50 e 59 anos (18,46\%), 13 mulheres entre 60 e 69 anos $(20,00 \%)$ e 15 mulheres com 70 anos e mais $(23,07 \%)$. Foi contabilizado um total de 50 de mulheres com neoplasias da pele $(77,53 \%)$ e 15 homens $(23,06 \%)$ dos 65 exames com diagnóstico de câncer de pele (Tabela 2).

Tabela 2. Incidência de diagnósticos de MC, CBC e CEC por gênero e idade nas biópsias com diagnóstico específico CPNM entre janeiro de 2015 e maio de 2016. Hospital do Câncer de Dr. José Figueiredo. Patrocínio / MG.

\begin{tabular}{lllllllllll}
\hline & $30 / 39$ anos & $40 / 49$ anos & \multicolumn{2}{l}{$50 / 59$ anos } & \multicolumn{2}{l}{$60 / 69$ anos } & \multicolumn{2}{l}{$70 / 79$ anos } \\
\hline Gênero & $\mathbf{n}$ & $\mathbf{\%}$ & $\mathbf{n}$ & $\mathbf{\%}$ & $\mathbf{n}$ & $\mathbf{\%}$ & $\mathbf{N}$ & $\mathbf{\%}$ & $\mathbf{n}$ & $\mathbf{\%}$ \\
\hline Masculino & 0 & 0,0 & 4 & 6,15 & 2 & 3,07 & 3 & 4,61 & 6 & 9,23 \\
\hline Feminino & 2 & 3,07 & 8 & 12,30 & 12 & 18,46 & 13 & 20,00 & 15 & 23,07 \\
\hline Resultados & 2 & 3,07 & 12 & 18,45 & 14 & 21,53 & 16 & 24,61 & 21 & 32,30 \\
\hline
\end{tabular}

Em relação à variável cor da pele todos os casos de MC ocorreram em pessoas brancas (não há nas biópsias informação quanto a classificação do fototipo de Fitzpatrick) em um total de 3. Para o CBC encontrou-se 29 mulheres e 10 homens com pele branca; 2

mulheres e nenhum homem de pele amarela. Entre os casos de CEC foram 14 mulheres e 3 homens de pele branca; 3 mulheres e 1 homem de pele amarela. Não há indivíduos de pele negra dentre as neoplasias de pele no estudo (Gráfico 2).

Gráfico 2.Cor da pele por diagnóstico de MC, CBC e CEC entre janeiro de 2015 e maio de 2016. Hospital do Câncer de Dr. José Figueiredo. Patrocínio/MG.

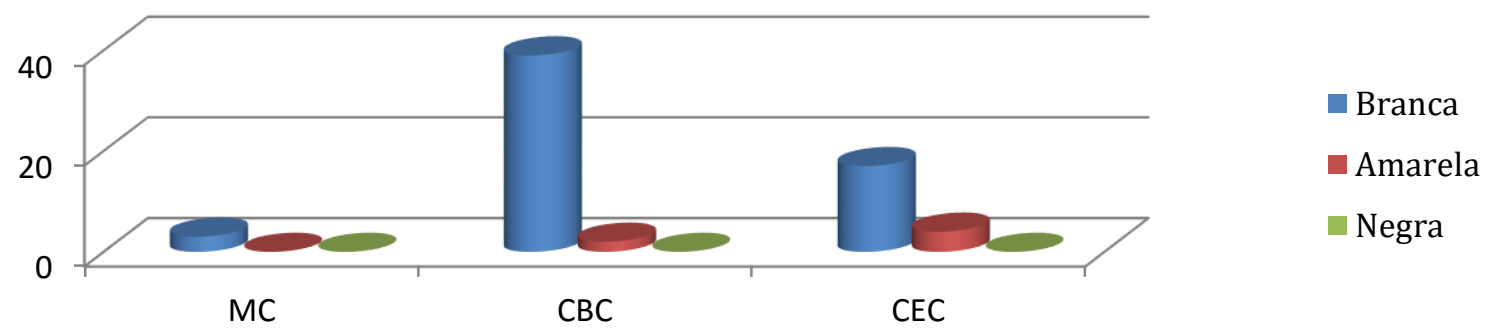


As localizações onde os tumores de pele diagnosticados como MC identificados são: 2 em membro inferior direito (MID) e 1 em região plantar do pé esquerdo; sexo feminino: MID (68 anos) e região plantar esquerda (44 anos); sexo masculino: MID (42 anos). Os tipos histológicos dos melanomas detectados foram: 2 MES (sexo masculino e feminino) e 1 MLA (sexo feminino) (Tabela 3).

Tabela 3.Tipo histológico, localização e gênero do MC das biópsias cutâneas avaliadas entre janeiro de 2015 e maio de 2016. Hospital do Câncer Dr. José Figueiredo. Patrocínio/MG.

\begin{tabular}{llll}
\hline Tipo histológico & & Localização & Gênero \\
\hline MES & $2^{*}$ & MID & M/F \\
MN & - & - & - \\
MLA & $1^{* *}$ & Região plantar e pé esquerdo & $\mathrm{F}$ \\
MLM & - & - & - \\
\hline
\end{tabular}

* Sexo: feminino, localização: MID, idade: 68 anos; Sexo: masculino, localização: MID, idade: 42 anos;

** Sexo: feminino, localização: plantar esquerda; idade: 44 anos.

\section{DISCUSSÃO}

O câncer de pele é o mais comum dentre as neoplasias malignas ${ }^{1,21}$. 0 melanoma é o tipo de neoplasia maligna cutânea menos comum, porém é a mais agressiva1,17,21. 0 MC é responsável por aproximadamente $79 \%$ das mortes por câncer de pele 22 . A quantidade de mortes provocadas por MC é considerada três vezes maior do que em todas as neoplasias de pele juntas ${ }^{10}$. As crescentes taxas de incidência e a alta mortalidade do melanoma cutâneo faz com que seja considerado o câncer de pele de maior importância21,22.

A incidência do MC e do CPNM encontradas no estudo, após o processamento e análise dos dados, apresenta muita proximidade com o esperado segundo as fontes consultadas. Depreende-se que a região onde ocorreu a pesquisa segue os padrões esperados para o MC e para o CPNM no Brasil. A submissão dos dados da pesquisa ao teste de Qui-quadrado de Pearson valida essa análise.

Considerando-se a variável gênero, a amostragem dos casos detectados de melanoma é considerada pequena (apenas 3 diagnósticos de MC). Encontra-se no Sistema de Informação sobre Mortalidade (SIM) por melanoma no ano de 2013 maior número de casos nos homens do que nas mulheres - dos 1547 óbitos são 903 homens e 644 mulheres $^{2}$.

0 MC é mais incidente em homens, porém no Brasil considera-se que essa variável é evidenciada com homegeneidade ${ }^{23}$.Azulay considera que as diferenças de incidência relacionadas ao gênero não são importantes. 0 prognóstico do MCM nas mulheres é considerado melhor em todos os estágios ${ }^{9}$.Ressalta-se que pode não ser prudente avaliar a característica gênero de forma concisa nesse trabalho, tratando-se de uma variável que é dependente da procura pelo serviço de saúde e das características da população estudada.

A cor da pele, para o MC, foi predominante no estudo com indivíduos brancos, apesar de não haver a classificação de Fitzpatrick nas biópsias analisadas, utilizando-se os critérios: branco, amarelo e negro. 0 fator de risco pele clara mostra-se preponderante para o MC1-11. Apesar de os outros tipos de pele também serem acometidos pele o melanoma, inclusive indivíduos de pele negra, esses achados são menos freqüentes ${ }^{9}$.

Para a variável idade é relatado que abaixo dos 40 anos o MC acomete mais pessoas do gênero feminino, já acima dos 50 anos a ocorrência é maior em indivíduos do gênero masculino ${ }^{16}$. Abaixo dos 40 anos o MC é incomum ${ }^{22}$.

Os estudos divergem em relação a idade predominante do aparecimento do MCM. Para Sampaio e Rivetti, encontra-se a idade predominante entre 30 e 60 anos $^{6}$. Para Hoff et al., a incidência maior está entre a quarta e sexta décadas de vida7. Estudo realizado em hospital do estado Santa Catarina entre 2003 e 2007 coloca o MC predominante na sexta década de vida ${ }^{17}$.

A localização do MCM é comum no tronco em homens e nos membros inferiores (MMII) 
entre as mulheres ${ }^{9,17}$. Detectados neste estudo, o MES é comumente detectado no dorso em homens e nas mulheres nos MMII10,23. Nesta pesquisa para o sexo masculino encontrou-se o MES no MID, contrariando outras pesquisas ${ }^{10,23}$. Para o MLA, que é frequente na região palmoplantar, os casos do estudo aqui apresentado se localizaram na região plantar esquerda, estando em acordo com outros estudos ${ }^{10,23}$.

Para o tipo histológico, MES é o tipo mais incidente, porém existe um estudo específico em uma localidade onde foi encontrado o $\mathrm{MN}$ mais incidente do que o MES15. Tal fato sugere que a amostragem estudada, a localidade, fatores socioeconômicos, o nível de exposição solar e a predisposição genética tem efeito sobre a população submetida ${ }^{15}$. A incidência do MC encontra-se em ascensão, dobrando o surgimento de casos a cada 10 ou 15 anos $^{24}$. Os fatores relacionados ao diagnóstico e prognóstico são de extrema importância na área oncológica. As biópsias submetidas à análise na pesquisa não fornecem detalhes padronizados como cor da pele, idade, classificação de Breslow e de Clark.

A profundidade do tumor é um fator fundamental para o prognóstico e tratamento, utilizados no estadiamento do $\mathrm{MC}^{25}$. É evidente que a padronização desses exames entre os laboratórios, profissionais e instituições da área oncológica tornam-se imprescindíveis para o manejo clínico e epidemiológico do câncer de pele. As Estimativas do INCA 2016 traz a informação da probabilidade de sub-registros devido ao subdiagnóstico do câncer de pele, a mais incidente neoplasia no Brasil ${ }^{1}$.

A educação em saúde, com o estímulo à proteção individual contra a RUV, é efetiva e tem custo financeiro baixo, sendo uma ação de prevenção primária do câncer de pele ${ }^{1,25}$. 0 acesso e arranjo dos serviços de saúde interferem na incidência do MC.A detecção do surgimento de novas manchas ou sinais na pele, com modificações na coloração, no tamanho e nas bordas de lesões antigas devem ser avaliadas ${ }^{1}$. É imprescindível a detecção precoce em estágios iniciais das neoplasias de pele, incluindo o melanoma pelo seu alto poder metastásico ${ }^{17,18}$.

Ressalta-se a importância de que mais estudos científicos precisam ocorrer na área oncológica. 0 câncer é considerado um problema de saúde pública no Brasil devido a sua magnitude epidemiológica, social e econômica ${ }^{3,18,24}$.

\section{CONCLUSÃO}

0 estudo concluiu que o MC é o menos incidente entre as neoplasias malignas da pele; que há predominância em pessoas de pele clara; variável gênero é dependente da população estudada; o MES é o tipo predominante; e que, a localização da lesão é pertinente com a pesquisas similares; mas, o fator idade mostra-se controverso.

A pesquisa propiciou informações sobre o câncer de pele numa região do estado de Minas Gerais e pode ser um sensor para a inclusão de ações de saúde sobretudo, nas de prevenção e de detecção precoce.

\section{REFERÊNCIAS}

1. Instituto Nacional do Câncer "José Alencar Gomes da Silva". Estimativa 2016: incidência de câncer no Brasil. Rio de Janeiro: INCA; 2015. 122p.

2. Instituto Nacional do Câncer "José Alencar Gomes da Silva". Pele melanoma [Internet]. Rio de Janeiro: INCA; 2017 [citado em: 20 jan 2017]. Disponível em: http://www2.inca.gov.br/wps/wcm/connect/tipos decancer/site/home/pele_melanoma/definicao+

3. Instituto Nacional do Câncer "José Alencar Gomes da Silva". ABC do câncer: abordagens básicas para o controle do câncer. Rio de Janeiro: INCA; 2011. 128 p.

4. Instituto Nacional do Câncer "José Alencar Gomes da Silva". Pele não melanoma [Internet]. Rio de Janeiro: INCA; 2017 [citado em: 26 jan 2017]. Disponível

em:

http://www2.inca.gov.br/wps/wcm/connect/tipos decancer/site/home/pele_nao_melanoma

5. Moreira RB, Schmerling RA, Buzaid AC. Algoritmo de manejo do melanoma cutâneo metastático. Rev Bras Oncol Clínica. 2014; 10(37):108-15.

6. Rivetti EA. Manual de dermatologia clínica de Sampaio e Rivitti. São Paulo: Artes Médicas; 2014.

7. Hoff PMG, organizador. Tratado de oncologia. São Paulo: Atheneu; 2013.

8. Wainstein AJA, Belfort FA. Conduta para o melanoma cutâneo. Rev Col Bras Cir. 2004; 31(3):204-14.

9. Azulay DR. Azulay: dermatologia. 6ed. Rio de Janeiro: Guanabara Koogan; 2013. 
10. Bogliolo L. Bogliolo patologia. Bogliolo Filho G, editor. 8ed. Rio de Janeiro: Guanabara Koogan; 2012. 11. Smeltzer SC, Bare BG. Brunner \& Suddarth: tratado de enfermagem Médico-cirúrgica. 13ed. Rio de Janeiro: Guanabara Koogan; 2015. v. 2.

12. Malagutti W, Kakihara CT. Curativos, estomia e dermatologia: uma abordagem multiprofissional. 2ed. São Paulo: Martinari; 2011.

13. Dimatos DC, Duarte FO, Machado RS, Vieira VJ, Vasconcellos VAC, Bins-Eli J et al. Melanoma cutâneo no Brasil. ACM Arq Catarin Med. 2009; 38(Supl. 1):14-9.

14. Sociedade Brasileira de Dermatologia. Classificação dos fototipos de pele [Internet]. Rio de Janeiro: Sociedade Brasileira de Dermatologia; 2017 [Citado em: 10 fev 2017]. Disponível em: http://www.sbd.org.br/cuidado/classificacao-dosfototipos-de-pele/

15. Araújo IC, Coelho CMS, Saliba GAM, Lana PC, Almeida ACM, Pereira NA, et al. Melanoma cutâneo: aspectos clínicos, epidemiológicos e anatomopatológicos de um centro de formação em Belo Horizonte. Rev Bras Cir Plást. 2014; 29(4):497503.

16. Ministério da Saúde (Br). Instituto Nacional do Câncer. Informativo detecção precoce: Monitoramento das ações de controle do câncer de pele. Rio de Janeiro: INCA. 2016; 7(3):1-8.[Citado em: 15 de fev de 2017] Disponível em:http://www1.inca.gov.br/inca/Arquivos/infor mativo_deteccao_precoce_03_2016.pdf

17. Purim KSM, Sandri CO, Pinto NT, Sousa RHS, Maluf EPC. Perfil de casos de melanoma em um hospital universitário, 2003 a 2007. Rev Bras Cancerol. 2013; 59(2):193-9.

18. Salvio AG, Assumpcao Junior A, Segalla JGM, Panfilo BL, Nicolini HR, Didone R. Experiência de um ano de modelo de programa de prevenção continua do melanoma na cidade de Jau-SP. An Bras Dermatol. 2011;86(4):669-74.

19. Instituto Brasileiro de Geografia e Estatística. Conheça cidades e estados do Brasil [Internet]. Rio de Janeiro: IBGE; 2017 [citado em: 16 fev 2017]. Disponível

em: https://cidades.ibge.gov.br/brasil/mg/patrocin io/panorama

20. Instituto Brasileiro de Geografia e Estatística. Índice de Desenvolvimento Humano. [Internet]. Rio de Janeiro: IBGE; 2010 [citado em: $17 \mathrm{fev}$ 2017]. Disponível

em: https://cidades.ibge.gov.br/brasil/mg/patrocin io/pesquisa/37/30255

21. American Cancer Society. Why you should know about melanoma [Internet]. Atlanta, Geórgia: ACS; 2017 [Citado em: 20 fev 2017] Disponível em: https://www.cancer.org/content/dam/cancerorg/cancer-control/en/booklets-flyers/why-youshould-know-about-melanoma-handout.pdf

22. Matheus LGM, Verri BHMAV. Aspectos epidemiológicos do melanoma cutâneo. Rev Ciênc Estud Acad Medicina. 2015(3):10-24.

23. Longo DL, organizador. Hematologia e oncologia de Harrison. 2ed. Porto Alegre: AMGH; 2015.

24. Pinheiro AMC, Friedman H, Cabral ALSV, Rodrigues HA. Melanoma cutâneo: características clínicas, epidemiológicas e histopatológicas no Hospital Universitário de Brasília entre janeiro de 1994 e abril de 1999. An Bras Dermatol. 2003;78(2):179-86.

25. Ministério da Saúde (Br). Secretaria de Atenção à Saúde. Protocolos clínicos e diretrizes terapêuticas em Oncologia. Brasília: Ministério da Saúde; 2014. $356 \mathrm{p}$.

CONTRIBUIÇÕES
Todos autores tiveram iguais
contribuições.

\footnotetext{
Como citar este artigo (Vancouver)

Silva RD, Dias MAI. Análise e incidência do melanoma cutâneo em um hospital de câncer localizado no Triângulo Mineiro. REFACS [Internet]. 2018 [citado em inserir dia, mês e ano de acesso]; 6(Supl. 1): 330-337. Disponível em: inserir link de acesso. DOI: inserir link do DOI.

Como citar este artigo (ABNT)

Silva, R. D.; DIAS, M. A. I. Análise e incidência do melanoma cutâneo em um hospital de câncer localizado no Triângulo Mineiro. REFACS, Uberaba, v. 6, p. 330-337, 2018. Supl. 1. Disponível em: <inserir link de acesso>. Acesso em: inserir dia, mês e ano de acesso. DOI: inserir link do DOI.

Como citar este artigo (APA)

Silva, R. D. \& Dias, M. A. I. (2018). Análise e incidência do melanoma cutâneo em um hospital de câncer localizado no Triângulo Mineiro. REFACS, 6(Supl. 1), 330-337. Recuperado em: inserir dia, mês e ano de acesso e, inserir link de acesso. DOI: inserir link do DOI.
} 